Original

\title{
Lipoatrofia semicircular: una revisión sistemática de la literatura
}

\section{Semicircular lipoatrophy: a sistematic review of the literature}

\author{
Rafael Díaz Sarmiento 1, 2, 3, Rafael Peña Rodríguez 1, 2, 4, Ángel Asúnsolo del Barco 2,5 \\ 1. Unidad docente Medicina del Trabajo. Castilla La Mancha \\ 2. Escuela Nacional de Medicina del Trabajo. Madrid. España \\ 3. Mutua Asepeyo \\ 4. Mutua Universal \\ 5. Universidad de Alcalá de Henares. Madrid. España.
}

Recibido: 30-01-11

Aceptado: 14-02-11

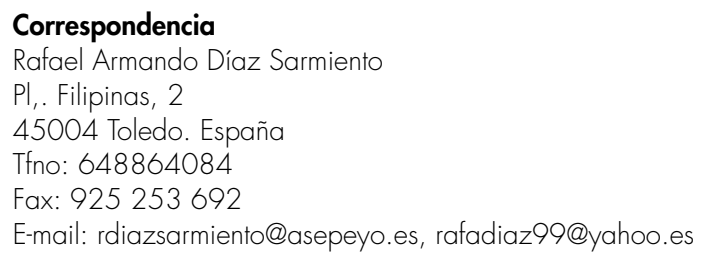

Resumen

Introducción: La lipoatrofia semicircular (LS) es un trastorno benigno del tejido subcutáneo, de causa desconocida. Se caracteriza por depresiones semicirculares en la cara anterolateral de los muslos, unilaterales o bilaterales. Se relaciona con condiciones ambientales laborales, microtraumas repetidos y factores personales. Se plateó esta revisión sistemática de la literatura científica para caracterizar el conocimiento existente.

Métodos: Revisión sistemática. Bases de datos: IBECS, IBSST, LILACS, IME, OSH-UPDATE, ISI-WOK, PubMed, CIS-DOC y Cochrane. Se incluyeron los artículos sobre lipoatrofia semicircular relacionada con condiciones de trabajo y medioambientales. Se utilizaron los niveles de evidencia científica del Scottish Intercollegiate Guidelines Network.

Resultados: Se recuperaron 66 artículos. Solo 22 cumplieron los criterios de inclusión: Quince con nivel de evidencia 3 y 7 con nivel 4. Siete estudios de series de casos y ocho de casos únicos, sumaron 838 casos, informados entre 1982-2010. Los principales factores de riesgo para LS son: Microtraumas repetidos, humedad relativa baja y electricidad en los edificios modernos de oficinas. Las depresiones semicirculares ocurren en la cara anterolateral de muslos, sin alteración de la piel, en mujeres (85\%), sin signos inflamatorios con histopatología inespecífica. La resonancia magnética, radiología y los anticuerpos antinucleares son negativos. El diagnóstico es clínico, apoyado con ecografía. El tratamiento psicológico y fisioterapia pueden ser útiles. Las medidas preventivas de mayor impacto son controlar los factores mencionados.

Conclusiones: Existe insuficiente evidencia científica sobre las causas de LS. Es un trastorno prevalente, que genera alarma, relacionado con las condiciones de trabajo. Es necesario continuar la investigación sobre agentes causales.

Med Segur Trab (Internet) 2011; 57 (222) 77-94

Palabras clave: lipoatrofia semicircular, factores de riesgo, síntomas, diagnóstico, tratamiento. 


\section{Abstract}

Introduction: The semicircular lipoatrophy (LS) is a benign disorder of the subcutaneous tissue with unkvown cause. It is characterized by unilateral o bilateral semicircular depressions in the anterolateral side of the thighs. It is related to environmental labor conditions, repeated microtraumas and personal factors. We conducted this systematic review of the scientific literature to characterize the existing knowledge.

Methods: Systematic Review. Databases: IBECS, IBSST, LILACS, IME, OSH-UPDATE, ISI-WOK, PubMed, CIS-DOC and Cochrane. We included articles about semicircular lipoatrophy related to working and environmental conditions. We used the levels of scientific evidence of the Scottish Intercollegiate Guidelines Network.

Results: 66 articles were recovered. Only 22 fulfilled the inclusion criteria: Fifteen with evidence level 3 and 7 with evidence level 4. Seven studies were series of cases and 8 were unique case reports. All of them added 838 cases, informed between 1982-2010. The principal risk factors for LS are repetitive microtrauma, low relative humidity and electricity in modern office buildings. The semicircular depressions occur in the anterolateral side of thighs, without alteration of the skin and muscles, mostly in women (85\%), without inflammatory signs and with unspecific histopathology. The magnetic resonance, $x$ rays and the antinuclear antibodies are negative. The diagnosis is clinical, supported by ultrasonography. Psychological treatment and physical therapy can be useful. The preventive measures of greater impact are controlling risk factors.

Conclusions: Insufficient scientific evidence exists about the causes of LS. It is a prevalent disorder that generates alarm, related to workplace conditions. It is necessary to continue investigating about causative agents.

Med Segur Trab (Internet) 2011; 57 (222) 77-94

Key words: Semicircular lipoatrophy, risk factors, symptoms, diagnosis, treatment. 


\section{INTRODUCCIÓN}

La lipoatrofia semicircular (LS) corresponde a una atrofia del tejido adiposo situado en el tejido subcutáneo, que ha sido descrita en diferentes localizaciones como muslos o antebrazos $^{1}$. Se caracteriza por depresiones circulares en banda ubicadas más frecuentemente en la semicircunferencia anterolateral del muslo, unilaterales o bilaterales. No afecta a tejidos suprayacentes e infrayacentes como piel y músculos, en la gran mayoría de los casos no produce síntomas, su evolución clínica es benigna, no produce secuelas y los casos remiten en un periodo de meses a años, al cesar la exposición a los factores de riesgo identificados.

Su prevalencia se ha establecido en 25 - 37\% en trabajadores de oficina ${ }^{2}$, siendo más frecuente en mujeres en la tercera o cuarta década de la vida. Aunque no es exclusiva del sexo femenino, algunos autores informan que la razón de ocurrencia de LS en mujeres y hombres de $6: 1^{3}$.

La LS fue descrita inicialmente por Gschwandtner y Münzberger, en Innsbruck, en 1974 4 . Posteriormente, en 1981, dos dermatólogos del St. Batholomew's Hospital en Inglaterra, hicieron una descripción de casos $^{5}$. A partir del 1995 se detectó un brote de 1300 casos en trabajadores de un banco en Bélgica ${ }^{2}$. Se han reportado de forma progresiva, casos en Francia e Italia.

Los primeros casos de LS en España se detectaron entre 2007 y 2008, en un brote de 1137 casos en varias empresas de oficinas de Barcelona $^{6}$, a partir de los cuales se inician las actuaciones de estudio, seguimiento y control por parte de la administración laboral y sanitaria, con el objetivo de investigar los factores de exposición que podían influir en el desarrollo de la lesión y proponer las medidas preventivas y correctoras en los centros de trabajo.

No se conocen las causas de la LS, se relaciona con alteraciones circulatorias, riesgo sanguíneo disminuido en las variantes de la arteria femoral lateral, microtraumas repetidos en muslos por los bordes de las mesas de trabajo, la presión de la silla en la cara posterior de los muslos, el uso de ropa compresiva, los campos electromagnéticos y la de electricidad estática. Sin embargo, las causas de la LS no han sido establecidas por métodos epidemiológicos, clínicos, bioquímicos, inmunológicos ni histológicos. Ante esta situación, se hace necesario actualizar y mejorar los niveles de evidencia científica acerca de las posibles causas de la LS y su prevención.

\section{OBJETIVO}

Caracterizar el estado del conocimiento sobre los factores de riesgo, aspectos clínicos y medidas preventivas de la lipoatrofia semicircular de origen ocupacional y medioambiental a partir de la literatura científica.

\section{METODOLOGÍA}

A continuación se describe la metodología utilizada en esta revisión bibliográfica.

\section{Búsqueda de la literatura científica}

Se realizó una búsqueda de la literatura científica disponible en las revistas y publicaciones indexadas en las bases de datos más utilizadas entre noviembre y diciembre de 2010. No se estableció ningún límite por fecha de publicación, edad y sexo.

Las bases de datos de revistas biomédicas y otras publicaciones científicas revisadas fueron: IBECS (Índice Bibliográfico Español en Ciencias de la Salud), IBSST (Índice Bibliográfico de Salud y Seguridad en el Trabajo), LILACS (Literatura científica y técnica de América Latina y del Caribe), IME (Índice Médico Español), OSH UPDATE (Occupational Safety and Health Update), ISI WOK (Institute for Scientific Information Web of Knowledge), 
PubMed (U.S. National Library of Medicine), CIS-DOC (International Occupational Safety and Health Information Centre -CIS- database) y The Cochrane Library.

La búsqueda en IBSST, LILACS, IBECS e IME se realizó con los siguientes términos: "Lipoatrofia semicircular", "Semicircular lipoatrofia”, Lipoatrofia, Lipodistrofia, Semicircular, Atrofia [DeCS] y lípidos [DeCS] y trabajo [DeCS]. La búsqueda en OSH UPDATE, WOK, PUBMED, COCHRANE y CIS-DOC: se realizó con los siguientes términos: Lipoatrophy, Lipodistrophy, Semicircularis, "Lipoatrophy semicircularis", "Lipoatrophia semicircularis", "Semicircularis lipoatrophy" y "Atrophy"[Mesh] AND "Lipids"[Mesh] AND "Work"[Mesh].

\section{Criterios de inclusión y exclusión}

Para esta revisión de la literatura científica sobre LS, de acuerdo con el objetivo del estudio, se establecieron los criterios de inclusión y exclusión de las publicaciones recuperadas, según se presentan a continuación.

\section{Criterios de inclusión}

Para esta investigación, se establecieron como criterios de inclusión, los siguientes:

- Artículos en español e inglés.

- Según el tipo de diseño, se incluyeron estudios descriptivos, series de casos, casos y controles, cohortes, experimentales y documentos técnicos.

- Según las características de la exposición y del efecto, se incluyeron los artículos sobre lipoatrofia semicircular relacionada con las condiciones de trabajo y medioambientales.

- Según el tipo de publicación, se incluyeron los informes originales, revisiones e informes técnicos.

- Como único de criterio de calidad, se estableció que los artículos incluidos en la revisión, fuesen publicados en revistas indexadas en las bases de datos seleccionadas, en las que generalmente se realiza revisión por pares.

\section{Criterios de exclusión}

Para esta investigación, se establecieron como criterios de exclusión, los siguientes:

- Artículos sobre lipoatrofia por terapias subcutáneas (insulinoterapia, metotrexato en lupus).

- Artículos sobre lipoatrofia de origen medicamentoso (antirretrovirales).

- Artículos sobre lipodistrofia congénita (síndrome de Berardinelli-Seip, mutación del gen heterocigoto LMNA y otras).

- Artículos sobre lipodistrofia de origen autoinmune (paniculitis lúpica).

- Artículos sobre lipodistrofia por causas infecciosas (Borrelia burgdorferi).

- Artículos sobre lipodistrofia de origen idiopático.

- Artículos sobre lipodistrofia asociada a alteraciones óseas.

- Artículos sobre LS no recuperados a texto completo.

\section{Métodos de combinación de los hallazgos}

El método de combinación de los hallazgos de las publicaciones incluidas en la revisión, que se aplicó por los dos investigadores, fue del tipo cualitativo, extractando de cada uno de los artículos, a manera de resumen, los aspectos relevantes para los diferentes aspectos presentados en el apartado de resultados: factores de riesgo, cuadro clínico, diagnóstico, tratamiento y medidas preventivas. 


\section{Niveles de evidencia de los resultados de los estudios incluidos en la revisión}

De acuerdo con los objetivos de esta revisión bibliográfica, la valoración del nivel de evidencia científica de los artículos sobre factores de riesgo y aspectos clínicos, se realizó según los criterios de la Scottish Intercollegiate Guidelines Network (SIGN) como se presentan en la tabla 1. Para los artículos sobre medidas preventivas se tuvieron en cuenta los grados de recomendación SIGN, como aparecen en la tabla 2.

\section{Tabla 1. Niveles de evidencia cientifica SIGN}

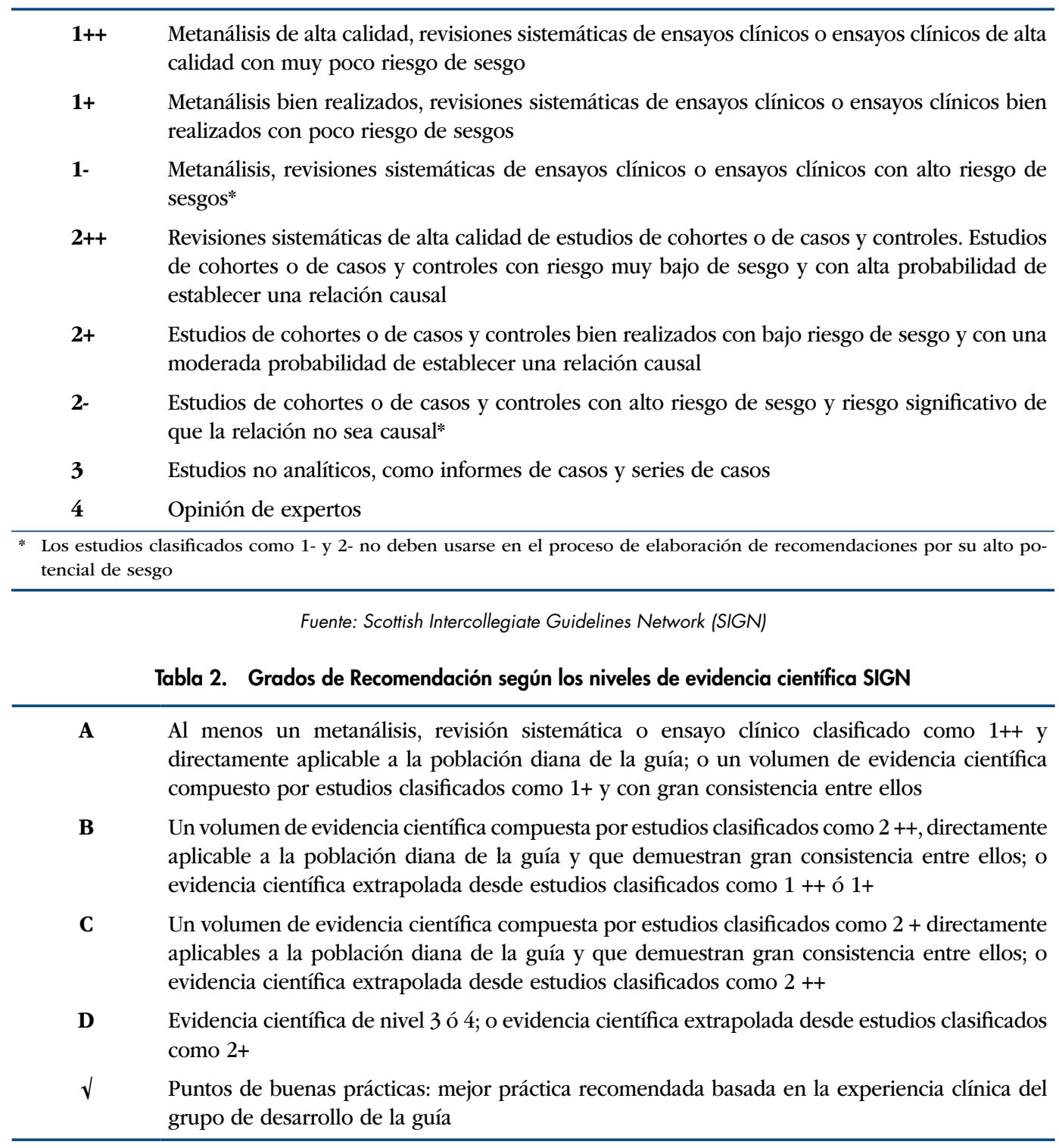

Fuente: Scottish Intercollegiate Guidelines Network (SIGN)

\section{RESULTADOS}

\section{Búsqueda de la literatura científica}

Como resultado de la estrategia de búsqueda se recuperaron 66 artículos. Tras eliminar repeticiones y aplicar los criterios de inclusión y exclusión se seleccionaron 22 artículos. Estos 22 artículos se revisaron a texto completo y se analizaron según los criterios de evidencia SIGN. Veintisiete artículos fueron excluidos. Los aspectos principales 
de los 22 artículos incluidos se presentan en la tabla 3. Los resultados de la revisión, se presentan en los apartados siguientes.

\section{Descripción de los estudios incluidos}

A continuación se presenta una breve descripción de las características de los 22 estudios incluidos en la revisión de acuerdo con el tipo de diseño y nivel de evidencia científica SIGN.

Los siete estudios de series de casos y los ocho casos reportados e incluidos en la revisión, suman 838 casos de LS, informados desde 1982 a 2010.

\section{Series de casos (Nivel de evidencia 3)}

En total se incluyeron en la revisión 7 estudios descriptivos o series de casos. Sus características principales se presentan a continuación.

Hermanns $\mathrm{V}$ et $\mathrm{al}^{7}$ evaluaron 220 casos de 1200 trabajadores de un edificio de oficinas. En una segunda etapa, realizaron un estudio de casos y controles con 21 trabajadores, 11 de ellos con LS, para determinar si la postura del cuerpo, el tipo de silla y la inclinación hacia delante, influyen en la LS. El nivel de evidencia para esta parte del estudio fue 2-.

Rodríguez MJ et $\mathrm{al}^{8}$, examinaron 153 trabajadores con sospecha de LS, remitidos de 27 empresas, incluyeron anamnesis clínica y laboral, riesgos extralaborales, medición de las lesiones en longitud, anchura, altura del surco y la distancia desde el suelo hasta el surco lesional con el trabajador calzado.

Rex J et $\mathrm{al}^{9}$, informaron una serie de dos casos clínicos de LS, en los que describen los aspectos clínicos y factores de riesgo.

Gruber PC y Fuller LC $^{10}$, describieron una serie de seis casos de LS en individuos que trabajaron en la misma oficina.

Senecal $\mathrm{V}$ et $\mathrm{al}^{11}$ presentan una serie de 18 casos de LS en una misma compañía. Cuatro de estos casos fueron evaluados en el servicio de salud laboral de la empresa.

Nagore E et al ${ }^{12}$ informan una series siete casos de LS, no relacionados entre ellos.

Pérez A et $\mathrm{al}^{6}$ presentan un estudio descriptivo, en el cual evalúan el impacto de las medidas de intervención, tomadas en 417 trabajadores con diagnóstico de LS desde febrero de 2007 hasta octubre de 2008, en varios centro de trabajo en Barcelona.

\section{Informes de un caso (Nivel de evidencia 3)}

En total se incluyeron en la revisión 8 informes de un caso. Sus características principales se presentan a continuación.

Guerrero A et $\mathrm{al}^{13}$, presentan el caso de una paciente con LS, se describió el cuadro clínico y el manejo realizado.

Zafra-Cobo MI et $\mathrm{al}^{3}$, presentan el caso de una paciente con LS, se describió el cuadro clínico y el manejo realizado.

Nogué $S^{14}$, describió un caso de lipoatrofia progresiva y localizada en sus extremidades inferiores.

Bordel-Gómez $\mathrm{MT}^{15}$, realizó el estudio del caso de una mujer que trabajaba en un invernadero, en la recogida de verduras, quien consultó por presentar lesiones deprimidas en la cara anterior del muslo izquierdo.

Ogino $\mathrm{J}$ et $\mathrm{al}^{16}$, reportan un caso de una mujer con surcos lineales en la cara anterior de ambos muslos, luego de llevar una faja elástica ajustada durante 2-10 horas al día, 3-4 días por semana desde diciembre 2002 a enero 2003, para corregir la forma de su cuerpo y tener las caderas más delgadas. Deja de llevar la faja después de notar los surcos, cuatro meses más tarde, no mejoró por lo que decide acudir a Ortopedia, es remitida a Dermatología 
y Radiología, para identificar posible causa traumática de LS. Realizaron diagnósticos diferenciales con panicultis del tejido conectivo, paniculitis lúpica y esclerodermia.

Herane MI et $\mathrm{al}^{17}$ reportan un caso de una mujer con LS.

Mascaro JM y Ferrando $\mathrm{J}^{18,19}$ reportan en dos publicaciones, dos casos de LS en hermanas usuarias de jeans (vaqueros ajustados).

\section{Documentos técnicos (Nivel de evidencia 4)}

En total se incluyeron en la revisión 7 documentos técnicos publicados. Sus características principales se presentan a continuación.

Pañella $\mathrm{H}$ et $\mathrm{al}^{20}$ elaboraron un documento técnico en el que describen cuadro clínico y medidas preventivas de la LS. Adicionalmente, relatan su experiencia en el manejo del brote de LS ocurrido en Barcelona en 2007, con 200 pacientes en un edificio nuevo, ocupado por 1000 trabajadores. No reportan datos del estudio descriptivo, ni del estudio de casos y controles.

En su documento sobre riesgos físicos emergentes, OSHA Europa ${ }^{21}$, resalta la LS en el ambiente de oficinas como un riesgo relativamente nuevo y cuyas causas son sujeto de controversia.

Cruells $\mathrm{JM}^{22}$, realizó una nota técnica sobre los factores de riesgo para la LS, el personal laboral más afectado, la valoración médica y las medidas preventivas a tomar en cuenta.

González A e Izaola $\mathbf{A}^{23}$, realizaron un documento técnico en el cual describen la clínica de la LS, los posibles agentes de riesgo, diagnóstico, diagnóstico diferencial, evolución, protocolo de actuación, evaluación de riesgos y medidas preventivas.

Pomares JM y Arrizabalaga $\mathrm{P}^{2}$, presentan un informe de expertos, en el cual reportan datos de la literatura y del brote epidémico de LS ocurrido en la empresa Gas Natural de Barcelona en el 2007, con 244 casos entre 900 trabajadores. Informan además, la identificación de más de 600 casos de LS en 25 empresas de Cataluña, Madrid y la Comunidad Valenciana.

Verschaeve L y Maes A. Presentan un ensayo in vitro ${ }^{24}$ y un informe técnico ${ }^{25}$ en el cual plantean la hipótesis de la estimulación eléctrica como causa de la LS.

\section{Factores de riesgo}

\section{Informes con nivel de evidencia 3}

Hermanns $\mathrm{V}$ et $\mathrm{al}^{7}$, no encontraron valores críticos de temperatura interior, campos electromagnéticos e iluminación como contribuyentes del problema. No se encontraron diferencias en cuanto a sexo, edad y peso corporal. Después del cambio de sillas por nuevos modelos ergonómicos, los signos desaparecieron en el 14\% de los afectados. Las evaluaciones de las sillas y las mesas usadas previamente cumplieron con los estándares ergonómicos (NEN 1812 y 2449).

En el estudio de casos y controles, Hermanns $\mathrm{V}$ et $\mathrm{al}^{7}$, encontraron que las personas con LS suelen sentarse en sillas muy altas $(46-52 \mathrm{~cm})$, con flexión del tronco hacia delante y no uso del soporte lumbar. Según electromiografía de los músculos del muslo, los trabajadores con LS presentaron más posturas estáticas en comparación con aquellos trabajadores sin LS. En cuanto la medición de la presión de la parte anterior silla sobre la cara posterior el muslo, esta fue mayor cuando el contacto fue 5 o más cm por arriba de la rodilla y menor cuando el contacto fue a nivel de la rodilla. Presiones menores a las anteriores, se encontraron en aquellos trabajadores usuarios de reposapiés. Esta presión aumentó en un $10 \%$, en las personas no usuarias de soportes lumbares o los que se sentaban con el tronco inclinado hacia delante. Un 30\% de aumento de presión se encontró en los trabajadores con posición estática (Nivel de evidencia 2-). 
Tabla 3. Artículos incluidos de la revisión sistemática de la literatura cientifica sobre lipoatrofia semicircular (1).

\begin{tabular}{|c|c|c|c|c|c|c|}
\hline Autor & Año & País & Tipo de estudio & Muestra & $\begin{array}{l}\text { Hallazgos } \\
\text { principales }\end{array}$ & $\begin{array}{c}\text { Nivel de } \\
\text { evidencia/ } \\
\text { Grado de } \\
\text { recomendación }\end{array}$ \\
\hline Pérez A & 2010 & España & $\begin{array}{l}\text { Evaluación de } \\
\text { intervenciones }\end{array}$ & 417 & $\begin{array}{l}\text { Efectividad de las } \\
\text { intervenciones estudiadas }\end{array}$ & $3 / D$ \\
\hline Rodríguez MJ & 2009 & España & Serie casos & 153 & $\begin{array}{l}\text { Factores de riesgos } \\
\text { Factores ambientales } \\
\text { Cuadro clínico } \\
\text { Historia clínica y laboral }\end{array}$ & $3 / D$ \\
\hline Verschaeve L & 2009 & Bélgica & Documento técnico & No aplica & $\begin{array}{l}\text { Hipótesis de estimulación } \\
\text { eléctrica }\end{array}$ & $4 / \sqrt{ }$ \\
\hline Verschaeve L & 2009 & Bélgica & Ensayo in vitro & No aplica & $\begin{array}{l}\text { Hipótesis de estimulación } \\
\text { eléctrica }\end{array}$ & $4 / \sqrt{ }$ \\
\hline Zafra-Cobo MI & 2008 & España & Caso clínico & 1 & $\begin{array}{l}\text { Cuadro clínico } \\
\text { Factores de riesgo }\end{array}$ & 3 \\
\hline Pañella $\mathrm{H}$ & 2008 & España & Documento técnico & No Aplica & $\begin{array}{l}\text { Cuadro clínico } \\
\text { Medidas preventivas }\end{array}$ & $4 / \sqrt{ }$ \\
\hline Guerrero A & 2008 & España & Caso clínico & 1 & Cuadro clínico & $3 / D$ \\
\hline Nogué S & 2008 & España & Caso clínico & 1 & $\begin{array}{l}\text { Factor ambiental } \\
\text { Factores de riesgo } \\
\text { Cuadro clínico } \\
\text { Historia clínica y laboral }\end{array}$ & $3 / D$ \\
\hline Pomares JM & 2008 & España & Documento técnico & No Aplica & $\begin{array}{l}\text { Factores de riesgo } \\
\text { Medidas preventivas }\end{array}$ & $4 / \sqrt{ }$ \\
\hline González A & 2007 & España & Documento técnico & No Aplica & $\begin{array}{l}\text { Factores de riesgos } \\
\text { Cuadro clínico } \\
\text { Historia clínica y laboral }\end{array}$ & $4 / D$ \\
\hline Herane MI & 2007 & Chile & Caso clínico & 1 & Cuadro clínico & $3 / \mathrm{D}$ \\
\hline Cruells JM & 2007 & España & Documento técnico & No Aplica & $\begin{array}{l}\text { Factores de riesgo } \\
\text { Valoración médica } \\
\text { Medidas Preventivas }\end{array}$ & $4 / D$ \\
\hline $\begin{array}{l}\text { Bordel-Gómez } \\
\text { MT }\end{array}$ & 2006 & España & Caso clínico & 1 & $\begin{array}{l}\text { Factores de riesgos } \\
\text { Cuadro clínico } \\
\text { Historia clínica y laboral. }\end{array}$ & $3 / D$ \\
\hline OSHA Europa & 2005 & Europa & Documento técnico & No aplica & Identificación del riesgo & 4 \\
\hline
\end{tabular}

Fuente: Revisión sistemática de la literatura

Tabla 3. Artículos incluidos de la revisión sistemática de la literatura científica sobre lipoatrofia semicircular (2).

\begin{tabular}{lcccccc}
\hline \multicolumn{1}{c}{ Autor } & Año & País & Tipo de estudio & Muestra & Hallazgos principales & $\begin{array}{c}\text { Nivel de } \\
\text { evidencia/ } \\
\text { Grado de } \\
\text { recomendación }\end{array}$ \\
\hline Ogino J & 2004 & Japón & Caso clínico & 1 & Factores de Riesgo & 3 / D \\
Cuadro Clínico \\
Hruber PC
\end{tabular}

Fuente: Revisión sistemática de la literatura 
Zafra-Cobo MI et $\mathrm{al}^{3}$, presentan el caso de una paciente, sin antecedentes médicoquirúrgicos de interés, con LS. La paciente utilizaba pantalones vaqueros ajustados cuyas arrugas al permanecer sentada, coincidían con las zonas afectadas. La paciente permanecía sentada con esta vestimenta durante mucho tiempo.

Rodríguez MJ et $\mathrm{al}^{8}$, encontraron que se relacionaba LS con ambientes diseñados o rediseñados (edificios modernos) con humedad relativa baja y cargas electrostáticas y electromagnéticas aumentadas; esta ultima debido al gran número de aparatos de ofimática y cableados existentes en el área laboral.

Nogué $\mathrm{S}^{14}$, encontró lesiones en los miembros inferiores, relacionadas con el cambio de mobiliario (más moderno) y la ubicación del trabajo en el puesto de trabajo.

Bordel-Gómez $\mathrm{MT}^{15}$, encontró que las lesiones desarrolladas por la trabajadora coincidieron con el lugar de apoyo de un cubo que empleaba para la recolección de verduras.

Rex $\mathrm{J}$ et $\mathrm{al}^{9}$, encontraron que el factor precipitante más probable de LS, fue el uso de mallas deportivas, utilizadas de manera habitual, coincidiendo la localización de aparición con la zona de máxima presión: tercio superior de la cara anterior de ambos muslos, a la misma altura.

Gruber PC y Fuller LC ${ }^{10}$, hallaron que los casos de LS se relacionaban con traumas repetidos con el borde superior de los escritorios. No encontró relación con la presencia de campos electromagnéticos, electricidad estática y baja humedad relativa.

En el caso informado por Ogino $\mathrm{J}$ et $\mathrm{al}^{16}$, el factor de riesgo más evidente fue el uso prolongado de la faja elástica por más de 8 horas al día, durante 3 - 4 días por semana.

Senecal V et al ${ }^{11}$ informaron que los casos aparecieron en una empresa de telecomunicaciones, con empleados jóvenes y dinámicos, recientemente trasladados a unas nuevas oficinas con diseño moderno y muebles de bordes afilados. Los empleados activos y con estrés, acostumbraban levantarse repetidamente y apoyarse en sus escritorios para intercambiar archivos con los colegas sentados enfrente. Todos los escritorios eran iguales: mesas de $70 \mathrm{~cm}$ de alto y $10 \mathrm{~mm}$ de espesor (Nivel de evidencia 3).

Herane MI et $\mathrm{al}^{17}$ reportan un caso de una mujer con LS quien trabajaba permanentemente sentada y que casi siempre vestía pantalones, los pliegues de los pantalones coincidían con las bandas cutáneas, por lo cual los autores plantean la hipótesis de que la constitución adiposa de los muslos de las mujeres, predispone a que la presión mecánica causada por vestir pantalones mientras se está sentado por largos períodos de tiempo, altera la circulación en estos tejidos y origina la LS.

\section{Informes con nivel de evidencia 4}

De acuerdo con Pañella $\mathrm{H}$ et $\mathrm{al}^{20}$, las hipótesis más aceptadas son microtraumas repetidos como los producidos por la presión contra sillas, tableros de hierro, lavabos y bordes de las mesas de trabajo. Otros factores de riesgo planteados son: el uso de pantalones vaqueros ajustados, bajo riego sanguíneo por malformación congénita de la arteria circunfleja femoral lateral, aunque es rara. En los edificios en que se presentaron los casos estudiados, existía sistemas de climatización, baja humedad relativa en el entorno de trabajo, factores contribuyentes a la existencia de altos niveles de electricidad estática. Los autores proponen que el mecanismo de lesión es la transferencia de electrones al apoyarse, en posición de bipedestación, en las mesas cargadas con electricidad estática. En referencia al estudio de casos y controles realizado presentan como factores de riesgo el borde de la mesa de trabajo delgado y angular y la resultante mayor intensidad de la transferencia de energía concentrada en una superficie fina (Nivel de evidencia 2-).

En la nota técnica elaborada por Cruells $\mathrm{JM}^{22}$, informan que los factores de riesgo se relacionan directamente con los edificios de oficinas nuevos y modernos, así como con los nuevos entornos de trabajo. Estos factores son: los microtraumas repetitivos (presión contra el mobiliario, piernas cruzadas, ropa ajustada), los campos electromagnéticos, la electricidad estática y la humedad relativa baja en la zona de trabajo. 
Pomares JM y Arrizabalaga $\mathrm{P}^{2}$, describen la LS como de origen multifactorial relacionada con microtraumatismos repetidos con objetos como mesas de canto fino, la compresión por ropa ajustada y la isquemia por compresión del tejido subcutáneo por la actividad muscular y los cambios posturales. Citan que una variante anatómica en la arteria circunfleja femoral lateral que nace en la arteria femoral, en lugar de la arteria femoral profunda, la cual aparece en el 3\% de la población general, en contraste con la prevalencia de LS descrita en el $25 \%$ de algunos lugares de trabajo. Otras hipótesis planteadas son los campos electromagnéticos generados por los equipos informáticos y el profuso cableado. Las descargas electrostáticas producidas por la diferencia de potencial entre el cuerpo y el borde la mesa, pueden dañar la estructura del tejido adiposo. Se ha observado que el aumento de la humedad ambiental, mejora la LS por lo cual se refuerza la hipótesis de origen electrostática, pues la humedad favorece la circulación de la electricidad y la reposición equilibrada de las cargas eléctricas.

Sobre el brote ocurrido en Barcelona, Pomares JM y Arrizabalaga $\mathrm{P}^{2}$ reportan que el examen higiénico ambiental de campos electromagnéticos fue negativo, sólo encontraron electricidad de 30 voltios en la estructura metálica de las mesas, muy por debajo de lo establecido en el ámbito técnico y legal. La humedad relativa fue del 30\% (Recomendado 40-50\%). Se encontró carga electrostática positiva en las personas, variable según calzado, vestuario y grado de humedad. Los bordes de las mesas eran por su diseño, finos y agudos. En este brote identificaron que los factores relacionados con los casos de LS fueron: la forma del borde la mesa, el número de apoyo o contactos con esta, la humedad relativa baja y la electricidad estática alta.

Verschaeve $\mathrm{L}$ y Maes $\mathrm{A}^{24,25}$ plantean que los aparatos eléctricos, la conductividad eléctrica de los escritorios, la presencia de iones eléctricos en el aire y la humedad relativa baja, son factores siempre presentes en los casos de LS. En un ensayo in vitro, encontraron daños en el DNA de los adipocitos sometidos a corriente eléctrica (prueba del cometa alcalino), lo cual puede predisponerlos a la fagocitosis por los macrófagos y los glóbulos blancos. Iguales daños en el DNA obtuvieron al someter las células sanguíneas de los pacientes con LS, lo que no ocurre en los trabajadores sin LS.

González A e Izaola A ${ }^{23}$, describen los posibles agentes causantes de LS: presión reiterada sobre el área afectada, usar de ropa compresiva, microtraumatismos acumulativos, presencia de campos electromagnéticos y electricidad estática.

La tabla 4 resume los factores relacionados con la aparición de LS.

Tabla 4. Factores de riesgo relacionados con lipoatrofia semicircular

\begin{tabular}{|c|c|c|c|}
\hline Factore & lacionados & Observaciones & Autores \\
\hline \multirow{3}{*}{$\begin{array}{l}\text { Condiciones } \\
\text { de los ambientes } \\
\text { de trabajo }\end{array}$} & Humedad relativa & $\begin{array}{l}\text { Sistemas de climatización, en edificios modernos } \\
\text { Humedad del } 30 \%(40-50 \%)\end{array}$ & $\begin{array}{l}\text { Rodríguez B } \\
\text { Cruells Mayor J } \\
\text { Pañellas H } \\
\text { Pomares JM } \\
\end{array}$ \\
\hline & $\begin{array}{l}\text { Campos } \\
\text { electromagnéticos }\end{array}$ & $\begin{array}{l}\text { Equipos de ofimática } \\
\text { Cableado profuso }\end{array}$ & $\begin{array}{l}\text { Hermanns V } \\
\text { Rodríguez B } \\
\text { Cruells Mayor J } \\
\text { Pomares JM } \\
\text { Verschaeve L } \\
\text { Pomares JM }\end{array}$ \\
\hline & $\begin{array}{l}\text { Electricidad estática } \\
\text { Electricidad baja } \\
\text { tensión }\end{array}$ & $\begin{array}{l}\text { Muebles metálicos } \\
\text { Equipos de ofimática } \\
\text { Cableado profuso } \\
\text { Moquetas / ropa de trabajo }\end{array}$ & $\begin{array}{l}\text { Rodríguez B } \\
\text { Cruells Mayor J } \\
\text { Verschaeve L } \\
\text { Pañellas H } \\
\text { Pomares JM } \\
\end{array}$ \\
\hline \multirow{3}{*}{$\begin{array}{l}\text { Antecedentes } \\
\text { Personales }\end{array}$} & $\begin{array}{l}\text { Malformaciones } \\
\text { Congénitas }\end{array}$ & $\begin{array}{l}\text { Arteria circunfleja femoral lateral que nace en la arteria } \\
\text { femoral, en lugar de la femoral profunda }\end{array}$ & $\begin{array}{l}\text { Pomares JM } \\
\text { Pañella H }\end{array}$ \\
\hline & Edad & Tercera década de la vida & Gruber P.C. \\
\hline & Sexo & Femenino (relacionado distribución del tejido adiposo) & $\begin{array}{l}\text { Zafra-Cobo } \\
\text { Gruber P.C. }\end{array}$ \\
\hline
\end{tabular}




\begin{tabular}{|c|c|c|c|}
\hline \multicolumn{2}{|c|}{ Factores relacionados } & Observaciones & Autores \\
\hline \multirow{4}{*}{$\begin{array}{l}\text { Microtraumas } \\
\text { repetidos / } \\
\text { Presión mecánica }\end{array}$} & $\begin{array}{l}\text { Bordes escritorios y } \\
\text { mesas de trabajo }\end{array}$ & Mesas bordes delgados y angulares & $\begin{array}{l}\text { Bordel M } \\
\text { Gruber P.C } \\
\text { Cruells Mayor J } \\
\text { Pañella H }\end{array}$ \\
\hline & Número de apoyos & Contacto repetido con bordes afilados de las mesas & $\begin{array}{l}\text { Bordel M } \\
\text { Herane Ml } \\
\text { Pomares JM } \\
\text { Senecal }\end{array}$ \\
\hline & $\begin{array}{l}\text { Utilización de } \\
\text { prendas ajustadas }\end{array}$ & $\begin{array}{l}\text { Vaqueros } \\
\text { Mallas deportivas } \\
\text { Fajas elásticas }\end{array}$ & $\begin{array}{l}\text { Zafra-Cobo } \\
\text { Cruells Mayor J } \\
\text { Herane Ml } \\
\text { Pomares JM } \\
\text { Panellas H } \\
\text { Rex J } \\
\text { Ogino J } \\
\end{array}$ \\
\hline & $\begin{array}{l}\text { Posturas } \\
\text { prolongadas }\end{array}$ & $\begin{array}{l}\text { Piernas cruzadas } \\
\text { Sillas sin apoyo lumbares } \\
\text { No estiramientos de las piernas }\end{array}$ & $\begin{array}{l}\text { Hermanns } \\
\text { Zafra-Cobo } \\
\text { Herane Ml } \\
\text { Pomares JM } \\
\text { Cruells Mayor J }\end{array}$ \\
\hline
\end{tabular}

Fuente: Revisión sistemática de la literatura

\section{Aspectos clínicos}

\section{Cuadro clínico}

\section{Informes con nivel de evidencia 3}

En los casos observados por Hermanns $\mathrm{V}$ et $\mathrm{al}^{7}$, se presentaron depresiones de 2 - 4 $\mathrm{cm}$ en la piel de los muslos, localizadas a $15-20 \mathrm{~cm}$ por encima de la rodilla. El $27 \%$ de los signos desapareció espontáneamente, algunos paciente presentaron una segunda depresión cutánea cercana a la primera. El 2\% de los casos reportó dolor o tensión local, los otros pacientes manifestaron el problema como estético. Estos signos aparecen en forma bilateral (85\%), en su mayoría mujeres (87\%), con medias de estatura de 1.65 metros $(\mathrm{DE}=0.06)$, de índice de masa corporal d $24(\mathrm{DE}=4)$ y de edad de 39 años $(\mathrm{DE}=8)$.

Guerrero A et al ${ }^{13}$, presentan el caso de una paciente, quien tuvo lesiones de aparición progresiva, bilaterales, "en escalón", en la zona anterolateral de ambos muslos. Su dimensiones en largo y ancho fueron: $17 * 3 \mathrm{~cm}$ y $15 * 2 \mathrm{~cm}$ respectivamente. Las dos lesiones se hallaron a una altura de $73 \mathrm{~cm}$ del suelo. No hubo síntomas inflamatorios. La paciente no tenía antecedentes personales relacionados con LS. El tiempo de evolución del cuadro al momento del diagnóstico fue de 7 meses.

Zafra-Cobo MI et al ${ }^{3}$, presentan el caso de una paciente, sin antecedentes médicoquirúrgicos de interés, con depresiones horizontales en la cara anterolateral del tercio medio de los muslos, de $4-5 \mathrm{~cm}$ de ancho, de 18 meses de evolución, con piel suprayacente normal y sin signos inflamatorios.

En los casos observados por Rodríguez $\mathrm{MJ}^{8}$, presentaron lesiones en la región antero lateral en uno o en ambos muslos (96\%), de predominio en el sexo femenino. Lesiones en uno o en ambos antebrazos y en el abdomen. Anchuras de los surcos de lesión varia entre $1-10 \mathrm{~cm}$, con localización a una altura media desde el suelo con el calzado habitual, hasta el punto medio del surco lesional, entre $21-84 \mathrm{~cm}$, moda $72 \mathrm{~cm}$. No hubo antecedentes de enfermedades ni de tratamientos locales. Algunos casos refirieron molestias inespecíficas como disestesias, prurito, tirantez, sensación de presión, aumento de sensibilidad táctil o dolor leve.

En el caso del estudio por Nogué $\mathrm{S}^{14}$, las lesiones de LS aparecieron en la cara anterior del muslo, bilaterales, con predominio derecho, sin cambios cutáneos. 
En el caso de estudio por Bordel $\mathrm{M}^{15}$, la lesión de LS se evidenció en la cara anterior del muslo izquierdo. Sin lesiones inflamatorios, no acompañando de síntoma general.

En la serie de casos descrito por Rex $\mathrm{J}^{9}$, se evidenció que las lesiones se localizan a nivel del tercio superior de la cara anterior de ambos muslos, a una misma altura, caracterizada desde el punto de vista clínico como depresión semicircular a modo de bandas horizontales deprimidas de $1.5 \mathrm{~cm}$ de ancho, con aspecto normal de la piel suprayacente. De manera ocasional hubo sensación de pesadez en las piernas, no precedida de fenómenos inflamatorios ni sintomatología general.

Gruber PC y Fuller LC $^{10}$, describen las lesiones como bandas horizontales en la cara anterior de los muslos, bilaterales y unilaterales, a $74 \mathrm{~cm}$ del piso con la utilización del calzado habitual, desarrolladas de 2 semanas a 2 años después de haber iniciado el trabajo, sin sintomatología. La razón de casos encontrada entre mujeres y hombres fue 6:1. La edad de los casos fue de 20 a 54 años. La estatura varió de $1.55-1.78 \mathrm{~m}$ y el peso de $55-85 \mathrm{~kg}\left(\mathrm{IMC}=22-35 \mathrm{~kg} / \mathrm{m}^{2}\right)$.

Cruells $\mathrm{JM}^{22}$, describe la LS en las partes del cuerpo que se apoyan en el borde de las mesas cuando se está de pie, en los antebrazos cuando se apoyan en el borde de la mesa al utilizar el ratón. Pudiendo ser unilaterales o bilateral, de $5-20 \mathrm{~cm}$ de largo, de 2 cm de ancho y de 1 - $5 \mathrm{~mm}$ de profundidad, con piel y músculos intactos, de localización preferentemente en la región anterolateral del muslo. A veces, se presentan hormigueos, sensación de piernas pesadas, en general síntomas muy vagos y poco precisos.

Ogino $\mathrm{J}$ et $\mathrm{al}^{16}$, hallaron que los dos surcos presentados por la paciente eran simétricos, semicirculares, horizontales sobre el aspecto anterolateral de ambos muslos, la piel que cubría los surcos era normal en color y textura, no hubo dolor ni prurito. La palpación no reveló induración sobre sus muslos. El sitio y la forma de los surcos, estuvieron en correspondencia con el borde inferior de la faja utilizada.

Senecal V et al $^{11}$ presentan el caso de una mujer de 27 años, sin antecedentes personales de importancia, con depresión subcutánea en la cara anterolateral de ambos muslos, notados por la paciente a las 6 semanas del inicio de su trabajo. La piel suprayacente fue normal sin induración a la palpación. No hubo dolor, sensación de quemadura o fatiga. El recuento de células sanguíneas, rayos $\mathrm{X}$ de tórax, electrocardiograma $\mathrm{y}$ electromiografía fueron normales. La paciente consultó por motivos estéticos. Los otros 3 casos fueron mujeres, con edad entre 26 - 32 años, todas trabajadoras en escritorios y con depresiones en bandas similares, con piel suprayacente normal, bilaterales y simétricas en 2 casos. Una mujer se quejó de piernas pesadas desde hace varios años y otra notó incremento de la depresión al caminar. Con base en estos 4 casos y 14 más detectados, establecieron una incidencia del $37.5 \%$ en los trabajadores de escritorio, con una razón mujeres a hombres de 2:1. Las mujeres estuvieron alrededor de los 30 años y los hombres entre 26 - 56 años. Las lesiones midieron de $3-12 \mathrm{~cm}$ horizontalmente, $1.5 \mathrm{~cm}$ verticales y 1 - $10 \mathrm{~mm}$ de profundidad. La mayoría de los casos fueron asintomáticos con piel suprayacente normal, aunque 4 pacientes tuvieron celulitis. Un paciente estaba en tratamiento por hipercolesterolemia. No se identificaron antecedentes de terapias con insulina, corticoides o acupuntura ni algún tipo de trauma.

Herane $\mathrm{MI}$ et $\mathrm{al}^{17}$ reportan un caso de una mujer con LS con dos depresiones semicirculares en la cara anterolateral de los muslos, de $3-4 \mathrm{~cm}$ de ancho y $15 \mathrm{~cm}$ de largo, bilaterales, simétricas, paralelas, de 1 año de evolución.

\section{INFORMES CON NIVEL DE EVIDENCIA 4}

Pañella $\mathrm{H}$ et al ${ }^{20}$ describen la LS como lesiones raras, con depresión semicircular, unilaterales o bilaterales, en la cara anterior o anterolateral de los muslos. Es más frecuente en mujeres y su ubicación varía según la persona pero coincide con la altura estándar de las mesas de trabajo teniendo en cuenta el tacón habitual. Las lesiones son horizontales, de 2 - $4 \mathrm{~cm}$ de ancho.

La tabla 5 resume los principales aspectos del cuadro clínico de la LS. 


\section{Diagnóstico}

\section{Informes con nivel de evidencia 3}

Según Guerrero A et al ${ }^{13}$, el diagnóstico de LS es clínico, basado en la inspección y palpación, confirmando la coincidencia de las lesiones con zonas de apoyo donde pueda haber una conducción de electricidad estática y/o electromagnética. Debe considerarse en el diagnóstico diferencial, la esclerodermia, paniculitis lúpica y la insulinoterapia subcutánea.

Tabla 5. Aspectos clínicos de la lipoatrofia semicircular

\begin{tabular}{|c|c|c|c|}
\hline \multicolumn{4}{|c|}{ Depresión semicircular } \\
\hline Signos y síntomas & Descripción & Observaciones & Autor \\
\hline Localización & $\begin{array}{l}\text { Cara anterolateral } \\
\text { de muslos }\end{array}$ & Antebrazos ocasionalmente & $\begin{array}{l}\text { Hermanns, Herane, Guerrero, } \\
\text { Nogué, Bordel, Gruber, Cruells, } \\
\text { Senecal, Herane }\end{array}$ \\
\hline Largo & $3-20 \mathrm{~cm}$ & Horizontalmente & $\begin{array}{l}\text { Hermanns, Guerrero, Cruells, } \\
\text { Senecal, Herane }\end{array}$ \\
\hline Ancho & $1,5-5 \mathrm{~cm}$ & & $\begin{array}{l}\text { Hermanns, Guerrero, Zafra-Cobo, } \\
\text { Rex, Cruells, Senecal, Herane }\end{array}$ \\
\hline Profundidad & $1-10 \mathrm{~mm}$ & & Cruells, Senecal \\
\hline Altura & $73-84 \mathrm{~cm}$ & $\begin{array}{l}\text { Desde el suelo con zapatos } \\
(15-20 \mathrm{~cm} \text {, por encima de las } \\
\text { rodillas) }\end{array}$ & $\begin{array}{l}\text { Herane, Gruber } \\
\text { Rodríguez }\end{array}$ \\
\hline Simétricas & Sí & & Herane, Senecal \\
\hline Bilaterales & Sí & $85 \%$ de casos. A veces unilateral & $\begin{array}{l}\text { Herane, Hermanns, Rodríguez, } \\
\text { Gruber, Rex, Cruells, Senecal }\end{array}$ \\
\hline Unilateral & Raro & & Gruber, Bordel, Cruells \\
\hline Más de 1 ipsilateral & Sí & & Hermanns \\
\hline Asintomáticas & Frecuente & & Herane \\
\hline Piel suprayacente & Normal & Celulitis ocasional & Senecal \\
\hline Dolor local & Raro & $\begin{array}{l}2 \% \text { de casos. Disestesias, prurito, } \\
\text { tirantez, sensación de presión, } \\
\text { aumento de sensibilidad táctil }\end{array}$ & Hermanns, Rodríguez, Cruells \\
\hline Pesadez / Cansancio & Rara & & Rex, Cruells \\
\hline Evolución & $12-18$ meses & Del cuadro clínico & Zafra-Cobo, Herane \\
\hline Incubación & 2 semanas - 24 meses & Desde inicio del trabajo & Gruber, Senecal \\
\hline \multicolumn{4}{|c|}{ Otras características } \\
\hline Problema estético & Sí & Motivo de consulta & Hermanns, Senecal \\
\hline Sexo femenino & Frecuente & $87 \%, 6: 1,2: 1$ & $\begin{array}{l}\text { Hermanns, Rodríguez, Gruber, } \\
\text { Senecal, Herane }\end{array}$ \\
\hline Edad & 39 años & $\begin{array}{l}\text { Media edad, DE=8 } \\
20-54 \text { años } \\
26-32 \text { años } \\
\text { Hombres } 26-56\end{array}$ & $\begin{array}{l}\text { Hermanns } \\
\text { Gruber } \\
\text { Senecal }\end{array}$ \\
\hline Talla & $1.65 \mathrm{~m}$ & $\begin{array}{l}\text { Media talla, } \mathrm{DE}=0.06 \\
1.55-1.78 \mathrm{~m}\end{array}$ & $\begin{array}{l}\text { Hermanns } \\
\text { Gruber }\end{array}$ \\
\hline Peso & $55-85 \mathrm{~kg}$ & & Gruber \\
\hline IMC & 24 & $\begin{array}{l}\text { Media IMC. DE=4 } \\
22-35 \mathrm{~kg} / \mathrm{m}^{2}\end{array}$ & $\begin{array}{l}\text { Hermanns } \\
\text { Gruber }\end{array}$ \\
\hline Antecedentes personales & Negativos & $\begin{array}{l}\text { Hipercolesterolemia } \\
\text { VHB (+) en casos aislados }\end{array}$ & Senecal \\
\hline $\mathrm{DE}=$ desviación estándar & & & \\
\hline
\end{tabular}

Fuente: Revisión sistemática de la literatura

En el estudio histopatológico del caso de LS reportado por Zafra-Cobo MI et $\mathrm{al}^{3}$, asociado al uso de vaqueros ajustados, se halló atrofia parcial del tejido graso con adipocitos pequeños mezclados con normales. Entre estos, se observó escaso material hialino eosinófilo. Por inmunohistoquímica, los adipocitos fueron positivos para Vicentina 
y proteína S-100. También se hallaron escasos elementos histiocitarios positivos para CD68. La analítica completa de sangre, perfil tiroideo y los estudios inmunológico y de autoinmunidad fueron normales.

En los casos observados por Rodríguez $\mathrm{MJ}^{8}$, los diagnósticos fueron clínicos, basados en la historia clínica y laboral.

El diagnóstico de la trabajadora, llevado a cabo por Nogué $\mathrm{S}^{14}$, fue clínico, la ecografía mostró perdida del 58\% del tejido celular subcutáneo en el muslo derecho y del $21 \%$ en el muslo izquierdo. La resonancia magnética de las extremidades inferiores confirmó la atrofia focal circunscrita al tejido graso subcutáneo, no evidenciándose alteración en los grupos musculares subyacentes, ni sustitución de tejido graso por tejido fibroso o de estirpe.

Bordel-Gómez $\mathrm{MT}^{15}$, basó su diagnóstico en la historia clínica, no realizó estudios histológicos. Los estudios complementarios: analíticas completas de sangre, hormonas tiroideas, estudios inmunológicos y electrocardiogramas fueron normales.

Rex $\mathrm{J}$ et $\mathrm{al}^{9}$, basaron su diagnóstico en la anamnesis minuciosa y la exploración física. Las pruebas de anticuerpos antinucleares fueron normales. No se realizó biopsia cutánea.

Gruber PC y Fuller LC ${ }^{10}$, basaron su diagnóstico en la anamnesis minuciosa (historia clínica y laboral) y la exploración física. Los estudios de laboratorios rutinarios fueron normales. Se sugirió la biopsia de la lesión siendo declinada por los trabajadores. palpable.

Cruells $\mathrm{JM}^{22}$, refiere que el diagnostico de LS es clínico. La lesión debe ser visible y

Ogino $\mathrm{J}$ et $\mathrm{al}^{16}$, hallaron en sus casos, rayos $\mathrm{X}$ negativos. La resonancia de los muslos mostró la pérdida de tejidos superficiales subcutáneos adiposos, no había alteración del grosor de grasa subcutánea y la dermis deprimida mostró el mismo grosor que la dermis circundante. El músculo no mostró ninguna atrofia. Las analíticas de rutina fueron normales, una paciente era portadora del virus de la hepatitis B. No tenía antecedentes de enfermedades metabólicas, ni hormonales, ni alteración del tejido conectivo que se relacionase con la presencia de los surcos a nivel de la dermis.

En el caso reportado por Herane MI et $a^{17}{ }^{17}$, en la ecotomografía de las áreas afectadas de la paciente se halló depresión simétrica del tejido subcutáneo de ambos muslos, más acentuada en el lado derecho, con una pérdida del patrón de ecogenicidad normal y compartimentación. La lesión más baja, alcanzó un espesor mínimo de $8.1 \mathrm{~mm}$. Por arriba y debajo de las lesiones, el tejido subcutáneo adyacente tenía un patrón normal de 10.6 $-12 \mathrm{~mm}$.

Rodríguez MJ et $a^{8}$, dentro del protocolo de actuación recomiendan realizar fotografía de la lesión y una ecografía de partes blandas en la primera visita. Adicionalmente buscar historia de exposición a humedad relativa baja, campos electromagnéticos y cargas electrostáticas en las superficies de las mesas y sillas de trabajo.

\section{INFORMES CON NIVEL DE EVIDENCIA 4}

Pañella $\mathrm{H}$ et $\mathrm{al}^{20}$ reportan que las lesiones de LS histopatológicamente son inespecíficas, con inflamación de vasos sanguíneos y pérdida parcial o completa de tejido adiposo, el cual es reemplazado por colágeno.

Pomares JM y Arrizabalaga $\mathrm{P}^{2}$, refieren según su revisión, la anatomopatología muestra: infiltración linfocítica perivascular, disminución del volumen y número de adipocitos, que posteriormente son reemplazados por tejido conjuntivo. Esto apunta a la participación de un mecanismo inmunitario. Se cree que la estimulación eléctrica activa los macrófagos con liberación de citocinas como el factor tumoral alfa, que estimula la fagocitosis del adipocito. Finalmente, recomiendan la ecografía de superficie como apoyo al diagnóstico, sin especificar hallazgos típicos y reafirmando que el diagnóstico es básicamente clínico. 
González A e Izaola A, describen que el diagnóstico diferencial de LS se debe hacer con esclerodermia, tratamiento antirretroviral, roturas musculares e inyecciones repetidas subcutáneas o intramusculares con corticoides o insulina.

La tabla 6 resume los principales síndromes y enfermedades relacionadas con lipodistrofia para el diagnóstico diferencial de LS.

Tabla 6. Síndromes y enfermedades para diagnóstico diferencial de lipoatrofia semicircular

\begin{tabular}{l|l}
\hline \multicolumn{1}{c|}{ Causas } & \multicolumn{1}{c}{ Síndromes y enfermedades } \\
\hline Metabólicas & $\begin{array}{l}\text { Diabetes mellitus } \\
\text { Dislipidemia }\end{array}$ \\
\hline Congénitas & $\begin{array}{l}\text { Síndrome de Berardinelli-Seip } \\
\text { Mutación del gen heterocigoto LMNA }\end{array}$ \\
\hline Autoinmune & Esclerodermia \\
& Paniculitis \\
& Paniculitis lúpica \\
\hline Infecciosas & Borrelia burgdorferi \\
& VIH/SIDA (Secundaria a terapia antirretroviral) \\
\hline Terapias subcutáneas & Tratamiento con Metrotexate \\
& Insulinoterapia \\
\hline Otras & Lipodistrofia idiopática \\
\hline
\end{tabular}

Fuente: Revisión sistemática de la literatura

\section{Tratamiento}

\section{INFORMES CON NIVEL DE EVIDENCIA 3}

Según Guerrero A et $\mathrm{al}^{13}$, no existe tratamiento específico y las lesiones se resuelven espontáneamente luego de finalizar la exposición a los factores de riesgo. El caso debe declararse como un accidente de trabajo y ser remitido a la mutua correspondiente.

Zafra-Cobo MI et $\mathrm{al}^{3}$, enfocaron el tratamiento de la LS a la eliminación de los factores desencadenantes y reportan la resolución progresiva en un período que oscila entre 9 meses y 4 años.

Gruber PC y Fuller LC ${ }^{10}$, enfocaron la base del tratamiento de la LS a la eliminación del factor desencadenante. Al igual que Zafra-Cobo et $\mathrm{al}^{3}$, reportaron que la resolución progresiva ocurre entre 9 meses y 4 años.

Cruells $\mathrm{JM}^{22}$, informa que no se conoce tratamiento medico específico y que es importante adaptar las condiciones del trabajo y del entorno a los factores etiológicos que se conocen.

Pérez A et $\mathrm{al}^{6}$ recomiendan el apoyo psicológico para controlar la alarma de los trabajadores y la fisioterapia local de las lesiones.

\section{INFORMES CON NIVEL DE EVIDENCIA 4}

Pomares JM y Arrizabalaga $\mathrm{P}^{2}$, con base en el brote ocurrido en Gas Natural en Barcelona, apoyan las medidas tomadas: terapia psicológica y la fisioterapia como apoyo terapéutico.

\section{Medidas Preventivas}

A continuación, se presentan las medidas preventivas y su grado de recomendación por nivel de evidencia, resumidas en la tabla 7 .

\section{Informes con nivel de evidencia 3, grado de recomendación D}

Las medidas preventivas implantadas por Cruells $\mathrm{JM}^{22}$, fueron: valores de humedad relativa por encima del 50\%, evitar el cableado sin protección adicional cerca a las patas de las mesas de oficina, evitar cajoneras totalmente metálicas, los materiales de los 
muebles de oficinas deben tener efecto disipativo de la electricidad estática, usar preferible calzado de cuero y evitar los de goma o sintéticos, no apoyar los pies en las patas de la silla, buena hidratación personal, no apoyar los muslos en el borde de la mesa, evitar ropa de tejidos sintéticos, los cantos de las mesas deben ser anchos, de manera que la superficie de contacto sea grande, evitando los cantos vivos y delgados.

Pérez A et $\mathrm{al}^{6}$, informaron que con la instalación de tomas a tierra de las partes metálicas de las mesas, humidificadores ambientales para mantener la humedad relativa $>50 \%$ y listones o perfiles para aumentar la superficie de los bordes de las mesas, el $90 \%$ de los casos se resolvió en 6 meses y se controló el brote de LS en diferentes lugares de trabajo en Barcelona.

Informes con nivel de evidencia 4 , grado de recomendación $\sqrt{ }$.

Hermanns $\mathrm{V}$ et $\mathrm{al}^{7}$, recomiendan la utilización de sillas con asiento horizontal con la parte anterior inclinada hacia delante y que el asiento llegue a la altura del hueco poplíteo, con superficie acolchada. Para esta medida en particular, el grado SIGN es: No recomendable. También recomiendan dar a los trabajadores instrucciones para sentarse y el apoyo lumbar al sentarse para facilitar la relajación y el movimiento alternativo de los grupos musculares.

De acuerdo con Pañella $\mathrm{H}$ et $\mathrm{al}^{20}$, de las medidas preventivas implantadas, la más efectiva reportada es: el cambio de los bordes afilados de las mesas a redondeados y amplios (reducción de casos anuales de 60 - 70 a 2). Las medidas para reducir la electricidad estática, como el aumento de la humedad relativa por encima del $45 \%$, presentaron una moderada reducción de los casos.

\section{CONCLUSIONES}

La LS un trastorno endémico en los lugares de trabajo, que genera alarma en los trabajadores, relacionado con las condiciones de trabajo. Aparece con frecuencia en los edificios de oficinas de diseño moderno nuevos o remodelados. Las depresiones semicirculares ocurren en la cara anterolateral de muslos, sin alteraciones de piel o músculos, frecuentemente en mujeres, sin signos inflamatorios y con histopatología inespecífica.

La evidencia científica sobre las causas de la lipoatrofia semicircular es insuficiente, de niveles SIGN 3 y 4, correspondientes a estudios descriptivos e informes técnicos, por lo que es necesario continuar la investigación sobre agentes causales, con estudios observacionales y de intervención. Los estudios experimentales no son viables por razones éticas.

No obstante lo anterior, son tres los principales factores de riesgo identificados para LS, los cuales deben ser prevenidos desde el diseño y rediseño de los lugares de trabajo. En orden de prioridad, estos factores son: microtraumas repetidos, humedad relativa baja y la electricidad.

Los microtraumas repetidos ocurren generalmente con bordes de mesas delgados, filosos y metálicos; los hábitos y prácticas de los trabajadores, en cuanto a apoyarse en los bordes de las mesas de trabajo repetidamente durante la jornada laboral, incrementan el riesgo. El uso de ropa ajustada aumenta el riesgo de microtrauma o puede producirlo directamente.

En segundo lugar, humedad relativa baja ambiental, alrededor de $30-40 \%$, es un factor de riesgo para LS. Se recomienda la humidificación artificial de los ambientes de trabajo para mantener la humedad relativa por encima del $50 \%$, cuando sea necesario.

Finalmente, el otro factor de riesgo relacionado con la LS es electricidad estática y la baja tensión recibida al contacto directo con el mobiliario en los edificios modernos de 
oficinas. Por lo anterior las recomendaciones incluyen la colocación de puestas a tierra de equipos y muebles y evitar mobiliario metálico.

Una vez presentado un caso de LS relacionada con las condiciones de trabajo, este debe notificarse como un accidente de trabajo. El diagnóstico es clínico, apoyado con ecografía. No se recomienda realizar biopsias, resonancia magnética, rayos X, anticuerpos antinucleares ni análisis de laboratorio clínico adicionales. Existen síndromes y enfermedades con los que se debe realizar diagnóstico diferencial. El tratamiento incluye el retiro de la exposición, la fisioterapia y la terapia psicológica.

Tabla 7. Medidas Preventivas para lipoatrofia semicircular (Grado de recomendación D, ل $)$

\begin{tabular}{ll}
\hline \multicolumn{1}{c}{ Tipo de Medida } & \multicolumn{1}{c}{ Recomendación } \\
\hline Ambientales & Humidificación para mantener la humedad por encima de $50 \%$ \\
& Puestas a tierra para disminuir la cargas electrostáticas \\
\hline Ergonómicas & Utilizar sillas con asiento horizontal, inclinadas hacia delante y \\
& a la altura del hueco poplíteo, con superficie acolchada \\
& Mesas con bordes redondeados amplios y colocación de \\
& protecciones en zonas de apoyo \\
& Evitar cajoneras totalmente metálicas \\
& Utilizar calzados de cuero \\
& Evitar el cableado por debajo de las mesas \\
& No apoyar los pies en las patas de las sillas \\
& No apoyar los muslos en el borde de las mesas \\
& Buena hidratación personal \\
& Muebles con efecto disipativo de energía estática \\
\hline Formación & Instrucciones como sentarse, para facilitar la relajación y \\
e Información & movimientos alternativos de los grupos musculares \\
& Evitar el uso de tejidos sintéticos \\
\hline
\end{tabular}

Fuente: Revisión sistemática de la literatura

\section{REFERENCIAS BIBLIOGRÁFICAS}

1. Departament de Treball, Departament de Salut. Lipoatrofia Semicircular: Protocolo de Actuación. Barcelona: Generalitat de Catalunya; 2009.

2. Pomares JM, Arrizabalaga P. Lipoatrofia semicircular. Med Clin (Barc). 2008; 130(6): 213-5.

3. Zafra-Cobo MI, Yuste-Chaves M, Garabito-Solovera E, Santos-Briz A, Morán-Estefanía M, UnamunoPérez P. Lipoatrofia semicircular inducida por presión. Actas Dermosifiliogr. 2008; 99: 396-8.

4. Curvers B, Maes A. Lipoatrophia semicircularis: a new office disease? [Internet]. Bruselas: KBC Bank \& Insurance Group; 2003 [acceso 10 de enero de 2011]. Disponible en : http://www.esdproducts.eu/ userfiles/files/esdcataloog/ESD\%20Journal_Bart\%20Curvers.pdf.

5. Quijada M. Lipoatrofia semicircular, la nueva enfermedad de las oficinas. Gestión Práctica de Riesgos Laborales. 2008; 50: 58-9.

6. Pérez A, Nebot M, Maciá M, Panadés R; Collaborative Group for Evaluation of LS Outbreak Control Measures. An outbreak of 400 cases of lipoatrophia semicircularis in Barcelona: effectiveness of control measures. J Occup Environ Med. 2010; 52(7): 751-7.

7. Hermanns V, Hautekiet M, Haex B, Spaepen AJ, Van der Perre G. Lipoatrophia semicircularis and the relation with office work. Applied Ergonomics. 1999; 30: 319-24.

8. Rodríguez MJ, Madrid F. Lipoatrofia Semicircular de origen laboral. Arch prev riesgos labor. 2009 ; 12 (1): $14-8$.

9. Rex J, Ribera M, Bielsa I, Ferrándiz C. Lipoatrofia semicircular en los muslos. Actas dermosifiliogr. 2000; 91(6): 285-7.

10. Gruber PC, Fuller LC. Lipoatrophy semicircularis induced by trauma. Clin Exp Dermatology. 2001; 26: 269-71.

11. Senecal S, Victor V, Choudat D, Hornez-Davin S, Conso F. Semicircular lipoatrophy: 18 cases in the same company. Contact Dermatitis. 2000; 42(2): 101-2. 
12. Nagore E, Sánchez-Motilla JM, Rodríguez-Serna M, Vilata JJ, Aliaga A. Lipoatrophia semicircularis--a traumatic panniculitis: report of seven cases and review of the literature. J Am Acad Dermatol. 1998; 39(5Pt2): 879-81.

13. Guerrero A, Orpella X, Ramírez L, Schlaghecke. Lipoatrofia semicircular. Formación Médica Continuada en Atención Primaria. 2008; 15(8): 525.

14. Nogué S, Sanz P, Tomás X, Farrús X. Lipoatrofia Semicircular. Med Clin (Barc). 2008; 130 (9): 360.

15. Bordel-Gomez MT. Lipoatrofia semicircular unilateral. Piel. 2006; 21(8): 414-5.

16. Ogino J, Saga K, Tamagawa M, Akutsu Y. Magnetic Resonance Imaging of Semicircular Lipoatrophy. Dermatology. 2004; 209(4): 340-1.

17. Herane MI, Urbina F, Sudy E. Lipoatrophia semicircularis: A compressive lipoatrophy consecutive to persistent mechanical presurre. J Dermatol. 2007; 34(6): 390-3.

18. Mascaró JM, Ferrando J. The perils of wearing jeans; lipoatrophia semicircularis. Int J Dermatol. 1982; 21(3): 138-9.

19. Mascaró JM, Ferrando J. The perils of wearing jeans; lipoatrophia semicircularis. Int J Dermatol. 1983; 22(5): 333

20. Pañella H, Juanola E, De Peray JLl, Artazcoz L. Lipoatrofia semicircular: un nuevo trastorno de salud relacionado con el trabajo. Gac Sanit. 2008; 22(1): 73-5.

21. European Agency for Safety and Health at Work. Expert forecast on emerging physical risks related to occupational safety and health. Luxembourg: European Agency Publications; 2005.

22. Cruells JM. Lipoatrofia semicircular, emerging occupational disease? DYNA. 2007; 82 (8): 450-4.

23. González A, Izaola A. Lipoatrofia semicircular. Prevención. 2007; 182: 28-35.

24. Verschaeve L, Maes A. In vitro investigations related to the hypothesis that Lipoatrophia semicircularis find its origin in electro-stimulation. J Appl Toxicol. 2009; 29(6): 478-82..

25. Verschaeve L, Maes A. Support for the hypothesis that electro-stimulation is responsible for Lipoatrophia semicircularis. Med Hypothesis. 2009; 73(5): 802-6.

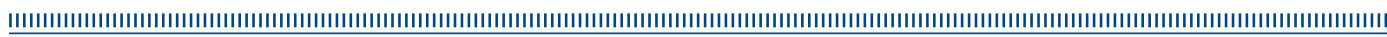

